\title{
Modelization of heat transfer and fluid dynamics in Solar Power Towers
}

\author{
J. Chiva' ${ }^{1}$ O. Lehmkuhl' ${ }^{2}$ M. Soria' ${ }^{1}$ A. Oliva ${ }^{1}$ \\ ${ }^{1}$ Centre Tecnològic de Transferència de \\ Calor (CTTC) \\ Universitat Politècnica de Catalunya (UPC) \\ ETSEIAT, C. Colom 11, 08222 Terrassa \\ (Barcelona), Spain \\ Fax: +34937398920 e-mail: cttc@cttc.upc.edu \\ 2 Termo Fluids S.L. \\ Magi Colet 8 \\ 08204 Sabadell, Barcelona, Spain \\ e-mail: termofluids@termofluids.com
}

\section{Introduction}

Solar thermal energy is becoming a very important renewable energy, with large scale plants operating in different parts of the world (NREL). The purpose of the current work is to describe the numerical model of a saturated steam solar receiver, that includes sub-models for heat conduction, two-phase flow, thermal radiation and turbulent natural convection using a Large Eddy Simulation (LES) method.

There are different designs for solar power collectors that are currently being used to generate electricity, like parabolic troughs, parabolic dishes and solar power towers. In a solar power tower, the solar radiation is reflected and concentrated by a field of mirrors into a receiver cavity. Inside this cavity, panels of tubes with circulating water receive this radiation and steam is produced. This steam is used to power turbines in order to generate electrical energy. The heat transfer and fluid dynamics phenomena in the receiver cavity are the interest of this study.

Termofluids (Lehmkuhl et al., 2007), a new general purpose unstructured and parallel object-oriented CFD code developed to solve industrial flows is used in this work.

\section{Heat conduction in the solid elements of the solar receiver}

Transient conduction heat transfer can be solved directly from the governing equation. For the case of transient heat conduction without volumetric sources, the energy transport equation can be written in integral form as:

$$
\left.\rho c_{p} \frac{\partial T}{\partial t}=\nabla \cdot(\lambda \nabla T) \quad \text { (eq. } 1\right)
$$

where $T$ represents temperature, $t$ is the time, $\rho$ is density, $c_{p}$ is the specific heat and $\lambda$ the thermal conductivity. Unfortunately, in the study case, due to the geometry of the receiver, a correct evaluation of conduction heat transfer is not straightforward. The size of the fins and tubes is of the order of millimeters while the overall receiver is of the order of meters. Therefore, large meshes have to be used to solve each panel, and parallel computers are needed. A non-structured mesh has been used, so that extensions to other geometries of the tubes can be implemented without substantial changes. A commercial program could have been used to generate meshes but, in order to reduce the time needed to change the density of the mesh or the number of pipes, a parametric mesh generator has been designed and implemented. This program allows us to obtain meshes with different densities and number of pipes and also, to concentrate the control volumes at the critical points (union between pipes and fins).

The finite control volume method has been used for the integration of the governing equations. A modified least-square method has been chosen for the calculation of the gradients. The equations are solved with the Conjugate Gradient method (Lehmkuhl et al., 2007).

The conduction model links all the other models through boundary conditions:

- Insulation at the back side of the panels: The back side of the panels of the receiver is covered with an insulating material. This situation is simulated using a one dimensional approach to obtain the 
value of the heat flux between the tubes and the air of the cavity:

$q=\alpha k \frac{(T w-T g)}{(k+\alpha \Delta x)}$ (eq. 2)

where $\mathrm{q}$ is the heat flux, $\alpha$ is the convective heat transfer coefficient, $k$ is the thermal conductivity of the insulator material, $T w$ and $T g$ are the temperature of the external wall of the tube and the temperature of the air respectively and $\Delta x$ is the thickness of the insulator.

- Solar radiation, thermal radiation and convection at the front side of the panels: The radiation reflected by the heliostats to the receiver cavity is almost completely absorbed by the front side of the panels. The radiometers situated in the receiver structure give a map of radiation distribution. This map is interpreted by the code to calculate the heat flux that enters the system by solar radiation (taking into account the absorptivity of the paint of the panels and the angle of incidence).

- The radiative heat transfer between the surfaces of the panels is calculated using the radiosity method. To reduce the computational cost, a simplified mesh is used to discretize the roof and the floor of the cavity, and the surfaces of the panels are considered isothermal when saw from each other.

- The convective heat transfer between the surfaces of the panels and the air of the cavity is calculated using the Newton's law of cooling :

$$
q=\alpha(T w-T g) \quad \text { (eq. 3) }
$$

and the convective heat transfer coefficient $\alpha$ is calculated from the Nusselt number (obtained as described in section 5):

$$
\alpha=\frac{k N u}{L} \quad \text { (eq. 4) }
$$

where $k$ is the thermal conductivity of the air and $L$ is the characteristic length.

- Forced convection inside the tubes: The convective heat transfer between the internal flow of the fluid and the tubes of the panels is calculated using Newton's law of cooling. The temperature of the fluid and the convective heat transfer coefficient are obtained from the two-phase flow model described in next section.

\section{Two-phase flow inside tubes}

The evaporation phenomena inside the tubes can not be solved directly from the governing equations (mass, energy and momentum conservation), because instantaneous tracking of the vapor-liquid inter-phase is too complex to do so. Therefore, in all the fields of science and engineering, no matter the computational resources available, two-phase flows in ducts must be solved using models.

In this work, a one dimensional quasi-homogeneous model has been chosen to represent the forced convection in the tubes, solved by the step by step method, which was used in the numerical analysis of twophase flow inside tubes (Morales Ruiz et al., 2009). The fact that this code is one dimensional makes difficult the communication between the two-phase flow code and the code for the solid elements. This problem has been solved calculating one heat transfer coefficient and one average wall temperature obtained from evaluating the energy conservation equation at the walls of the pipes.

For this model, a point of crucial importance is the critical heat flux (CHF), an abrupt rise of wall temperature due to a slight change in one of the flow variables. The temperature rise may be of two or three orders of magnitude, which most likely will cause a failure of the heated surface. Finding the CHF in terms of saturated evaporation is not straightforward. There is a wide variety of correlations that try to predict this condition and the corresponding location of the point of dry-out inside pipes, but there is not a correlation that provides satisfactory results under different geometries and working conditions of the fluid. So far, the 
Look-up tables (LUT) method seems to be one that can give similar results to the experiments. Moreover, this method allows to extend this application to different situations in a manner not too complicated. The implementation of this method in the code was made using the LUT of 2006 (Groeneveld et al., 2007). These tables are a function of pressure, mass flow and quality of steam. It is necessary to make a process of linear interpolation between the various tables that exist to find the CHF corresponding to the working conditions of the fluid.

The two-phase flow code has been validated with:

- Experimental data for low energy flow (Kattan et al., 1998; Cavallini et al., 2001; Jung and Didion, 1989).

- Experimental data obtained at the CTTC laboratory using a vapor compression refrigeration system (Rigola et al., 2003).

- Experimental data for high energy flow and correlations to predict the status of CHF (Groeneveld et al., 2007; Wong et al. 1990).

\section{Thermal radiation between the walls of the solar receiver cavity}

Every surface emits energy in the form of thermal radiation just by the fact that it is at a finite temperature. In the cavity of the solar receiver there is an exchange of energy between the surfaces (the ducts and fins of the panels, the exterior, the ceiling and the floor of the chamber) through thermal radiation. To calculate the radiative heat transfer between the surfaces of the cavity we propose to use the radiosity method (Modest, 1993). To use this method we make the following assumptions

- All the surfaces are diffuse, gray and opaque to thermal radiation.

- The medium is non participant.

- The opening of the cavity is considered as a black-body.

The radiosity represents the rate at which radiation leaves a unit area of the surface. This radiation could be intercepted by another surface and there is where the view factor between those surfaces could be of interest.

For the calculation of the view factor the 2LI (Double Line Integration) method (Walton, 2002) has been chosen. Gaussian integration method has been used to improve the accuracy and reduce the calculation time.

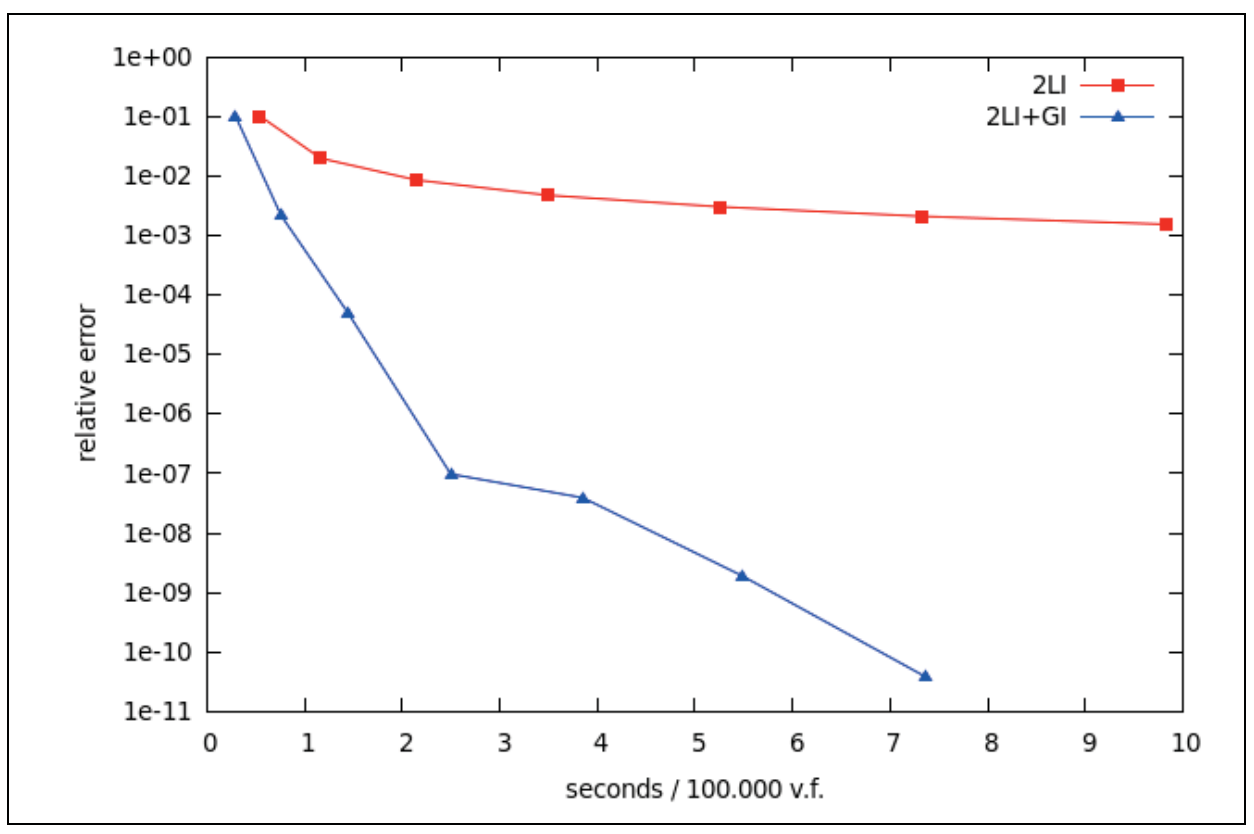

Fig. 1: Reduction of error using Gaussian Elimination. 
The singularity found when two surfaces are in contact has been solved using an analytic solution.

\section{Convection inside the cavity}

The temperature differences between the tubes and the air surrounding them causes heat transfer by convection. This heat transfer is complicated to evaluate numerically because:

- The geometry is complex and there are large differences between the size of the tubes (millimeters) and the cavity (meters).

- The cavity is open and interacts with the medium, so the domain has to be extended to include a part of the external environment.

- The air conditions in the real case (velocity and direction of wind, temperature, humidity, etc.) are variable in time and space.

To obtain a first approach, some assumptions has been made:

- The cavity has been simplified, the panels of tubes has been modeled as a hot isothermal wall and the rest of the surfaces has been supposed adiabatic:

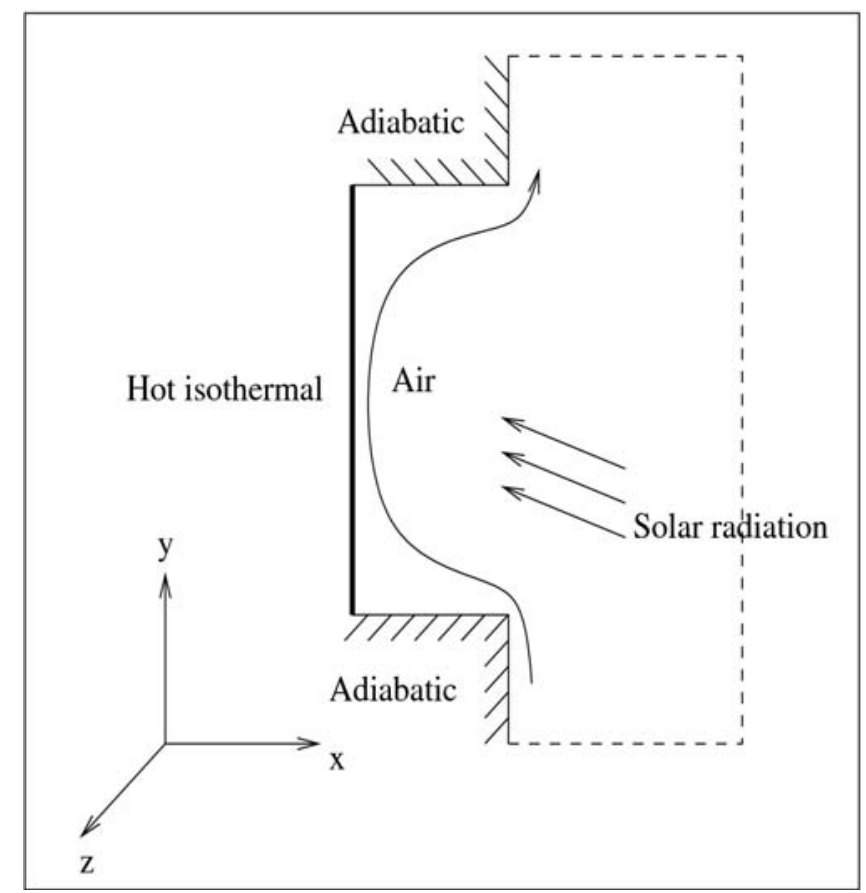

Fig. 2: Simplified cavity scheme.

- There is no wind (only natural convection) and the temperature of the air away from the cavity is constant.

- The air is supposed an incompressible Newtonian fluid, with constant thermophysical properties and negligible interaction in radiation. The Boussinesq approximation for buoyancy is also assumed.

Under this conditions, the governing Navier-Stokes equations for the conservation of mass, momentum and energy can be written as follows:

$$
\begin{aligned}
& \nabla \cdot \vec{u}=0 \quad \text { (eq. 5) } \\
& \frac{\partial \vec{u}}{\partial t}+(\vec{u} \cdot \nabla) \vec{u}=-\frac{1}{\rho} \nabla p_{d}+v(\Delta \vec{u})-\beta\left(T-T_{\text {ref }}\right) \vec{g} \quad \text { (eq. 6) }
\end{aligned}
$$




$$
\frac{\partial T}{\partial t}+\vec{u} \cdot \nabla T=\alpha \Delta T
$$

where $\vec{u}$ is the velocity vector, $\rho$ is the density, $p_{d}$ is the dynamic pressure, $v$ is the kinematic viscosity, $\vec{g}$ is the acceleration due to gravity, $\beta$ is the thermal expansion coefficient and $\alpha$ is the thermal diffusivity.

This problem is characterized by the Rayleigh number (Prandtl number is assumed constant to 0.71):

$$
R a=\frac{g \beta\left(T_{h}-T_{c}\right) L^{3}}{v \alpha} \text { (eq. 8) }
$$

where $L$ is the characteristic length (in the study case it is considered the height of the isothermal surface). Rayleigh numbers up to $10^{13}$ has been studied.

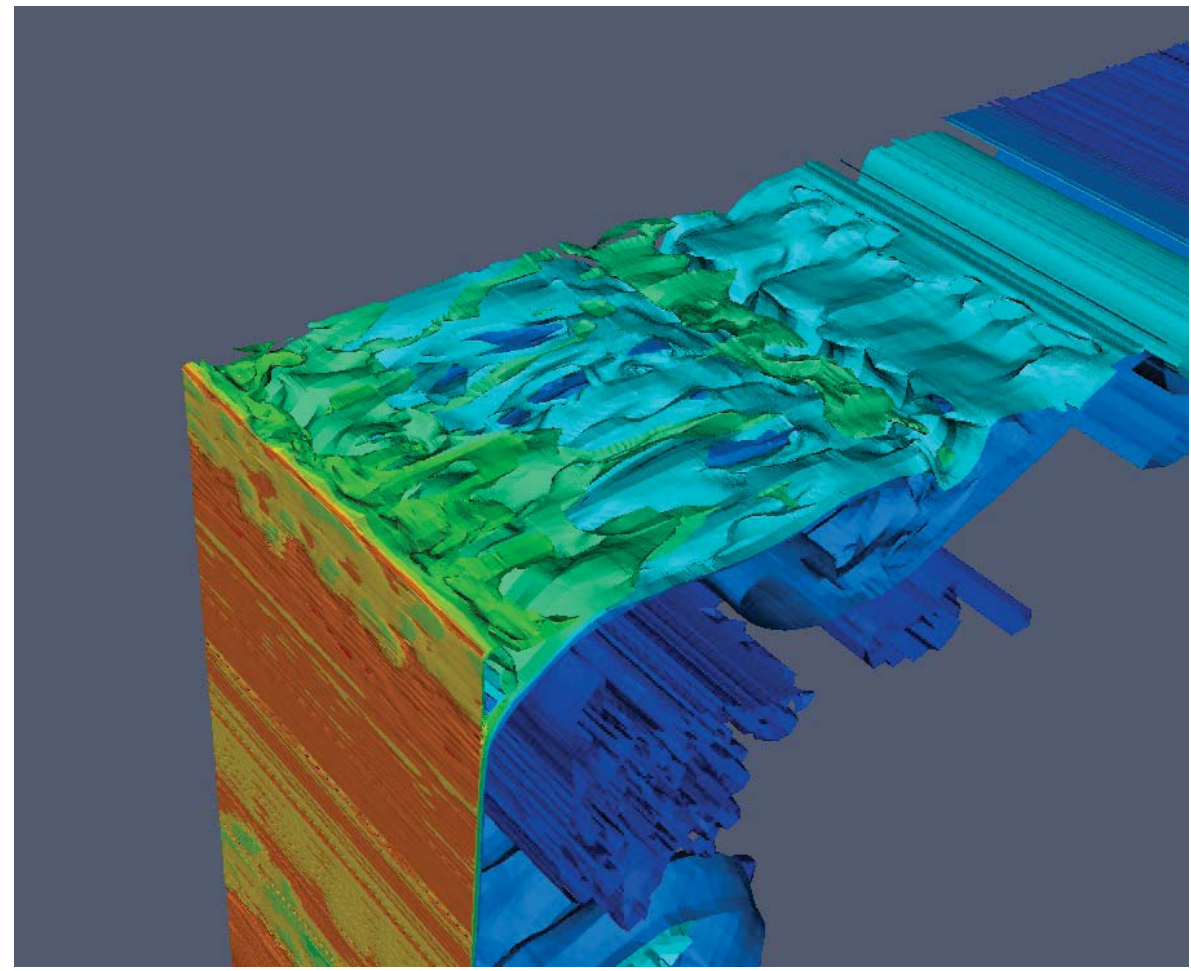

Fig. 3: Illustrative results for Rayleigh $=8^{12}$.

To reduce the computational costs, the cavity is assumed to be periodical in the $\mathrm{Z}$ axis, so only one slice of it is solved (this approach was used for instance in Trias et al., 2004, 2007).

The modeling of the turbulence has been done using the subgrid scale (SGS) model proposed by Yoshizawa et al. (2000).

With the simulation of this case, the heat transfer at the hot wall can be obtained as the local and average Nusselt number:

$$
\begin{aligned}
& N u=\frac{L}{T_{h}-T_{c}}\left(\frac{\partial T}{\partial x}\right)_{\text {Wall }} \text { (eq. 9) } \\
& \overline{N u}=\frac{1}{L} \int N u(y) d y \quad \text { (eq. 10) }
\end{aligned}
$$

and the Nusselt number can be used to calculate the heat transfer coefficient. A correlation between Rayleigh and average Nusselt numbers has been obtained and it will be used by the conduction model. 


\section{Conclusions}

A methodology to simulate heat transfer and fluid dynamics phenomena in the receiver cavity of a Solar Power Tower has been presented. Models dedicated to heat conduction in solids, two-phase flow, thermal radiation and natural convection have been described.

In future works, it would be interesting to investigate different fluids (over heated vapor, molten salts, etc.) as well as the effect of wind and other parts of a solar power tower plant.

\section{References}

Cavallini A., Censi G., Del Col D., Doretti L., Longo G.A. and Rossetto L., 2001. Experimental Investigation on Condensation Heat Transfer and Pressure Drop of New HFC Refrigerants (R134a, R125, R32, R410A R236ea) in a Horizontal Smooth Tube, Int. Journal of Refrigeration, vol. 24, pg. 73-87.

Groeneveld D.C., Shan J.Q., Vasic A.Z., Leung L.K.H., Durmayaz A., Yang J., Cheng S.C. and Tanase A., 2007. The 2006 CHF Look-up table, Nuclear Engineering and Design, vol 237, pg. 1909-1922.

Jung D.S. and Didion A., 1989. Electrical Power Research Institute (EPRI) ER- 6364, Research Project 8006-2.

Kattan N., Thome J.R. and Fravrat D., February 1998. Flow Boiling in Horizontal Tubes: Part I Development of a Diabatic Two-Phase Flow Pattern map, Journal of Heat Transfer, vol. 120, pg. 140-147.

Lehmkuhl O., Pérez Segarra C. D., Borrell R., Soria M. and Oliva A., 2007. TermoFluids: A new Parallel unstructured CFD code for the simulation of turbulent industrial problems on low cost PC Cluster. Proceedings of the Parallel CFD 2007 Conference, pg. 1-8.

Modest M. F., 1993. Radiative heat transfer, McGraw-Hill, Inc., New York.

Morales Ruiz S., Rigola J., Pérez Segarra C.D. and García Valladares O., 2008. Numerical analysis of twophase flow in condensers and evaporators with special emphasis on single phase-two phase transition zones. Applied Thermal Engineering 29, pg. 1032-1042.

NREL, National Renewable Energy Laboratory. Concentrating Solar Power Projects, http://www.nrel.gov/csp/solarpaces/

Rigola J., Pérez Segarra C.D., and Oliva A., 2003. Modelling and numerical simulation of the thermal and fluid dynamic behaviour of hermetic reciprocating compressors - part 2: Experimental investigation. Int. Journal of Heating Ventilating Air Conditioning and Refrigeration Research, 9(2), pg. 237- 250.

Trias F.X., Soria M., Pérez Segarra C.D. and Oliva A., 2004. Direct numerical simulation of a threedimensional natural convection flow in a differentially heated cavity of aspect ratio 4, Numerical Heat Transfer part A-Applications 45, pg. 649-673.

Trias F.X., Soria M., Pérez Segarra C.D. and Oliva A, 2007. Direct numerical simulations of two and three dimensional turbulent natural convection flows in a differentially heated cavity of aspect ratio 4, Journal of Fluid Mechanics 586, pg. 259-293.

Walton G.N., 2002. Calculation of Obstructed View Factors by Adaptive Integration. National Institute of Standards and Technology, NISTIR 6925. http:/www.bfrl.nist.gov/IAQanalysis/docs/NISTIR-6925.pdf.

Wong Y.L., Groeneveld D.C. and Cheng S.C., 1990. CHF prediction for horizontal tubes. Int. Journal of Multiphase Flow, 16, pg. 123-138.

Yoshizawa A., Kobayashi K., Kobayashi T. and Taniguchi N., 2000. A nonequilibrium fixed parameter subgrid-scale model obeying the near-wall asymptotic constraint, Physics of Fluids 12, pg. 2338-2344. 